\title{
Incentive Mechanism in Participatory Sensing for Ambient Assisted Living
}

\author{
Hu Yao, Wu Muqing and Li Tianze \\ Beijing Laboratory of Advanced Information Networks \\ Beijing Key Laboratory of Network System Architecture and Convergence \\ Beijing University of Posts \& Telecommunications, No.10, Xi Tu Cheng Road, Beijing, China \\ [e-mail: huyao800@gmail.com] \\ *Corresponding author: Hu Yao
}

Received Mach 24, 2017; revised July 27, 2017; revised August 30, 2017; accepted September 20, 2017; published January 31, 2018

\begin{abstract}
Participatory sensing is becoming popular and has shown its great potential in data acquisition for ambient assisted living. In this paper, we propose an incentive mechanism in participatory sensing for ambient assisted living, which benefits both the platform and the mobile devices that participated in the sensing task. Firstly, we analyze the profit of participant and platform, and a Stackelberg game model is formulated. The model takes privacy, reputation, power state and quality of data into consideration, and aims at maximizing the profit for both participant and publisher. The discussion of properties of the game show that there exists an unique Stackelberg equilibrium. Secondly, two algorithms are given: one describes how to reach the Stackelberg equilibrium and the other presents the procedures of employing the incentive strategy. Finally, we conduct simulations to evaluate the properties and effectiveness of the proposed mechanism. Simulation results show that the proposed incentive mechanism works well, and the participants and the publisher will be benefitted from it. With the mechanism, the total amount of sensory data can be maximized and the quality of the data can be guaranteed effectively.
\end{abstract}

Keywords: Ambient assisted living, participatory sensing, incentive mechanisms, game theory.

This work is supported by the 111 project (NO. B17007), by the Director Funds of Beijing Key Laboratory of Network System Architecture and Convergence (2017BKLNSAC-ZJ-06).

Part of the work was presented at the IEEE ICSESS'16 (Li Tianze, Wu Muqing and Wu Yuewei, "An game theory based online incentive mechanism for participatory sensing," 2016 7th IEEE International Conference on Software Engineering and Service Science (ICSESS), Beijing, 2016, pp. 700-705.). 


\section{Introduction}

Ambient assisted living (AAL) is an intelligent system of assistance, which enables a healthier and safer life in the preferred living environment. It integrates concepts, products, and services based on new technologies that interact with the environment, and aims at enhancing the quality of our life for all individuals at all life stages.

The main challenge for design an AAL system is a large amount of data which must be collected and processed for detecting events. The AAL system uses multiple sensors for data acquisition in order to obtain reliable data for later processing. After that, the system combines data from multiple sources to define the consumer's routine, detect unusual events, make the right decisions and perform the proper action in response [1], [2].

With the development of participatory sensing, there comes a good way for data acquisition. The new generation smart devices have multiple embedded sensors such as GPS, microphone, gyroscope, camera, accelerometer, digital compass, etc. [3]. The set of the available sensors can be considered as a private sensor network that connects the smart device with its physical environment, allowing a new kind of application, which recruits general public to collect and share sensory information, emerging on this trend is participatory sensing [4]. Using these embedded sensors, the general public act as participants to sense information from the surrounding environment and share the information using existing communication infrastructure [5]. Participatory sensing has been used for environmental monitoring, healthcare, social networks, and has great potential for ambient assisted living.

Different types of participatory sensing applications are proposed and used in our daily life for ambient assisted living. For example, a participatory sensing platform named APISENSE helps scientists to collect realistic data about some disease from a population of voluntary participants [6]. The U.S. Food and Drug Administration (FDA) approves the use of smartphones for the collection of medical data through participatory sensing [7]. The key features and challenges of participatory sensing are surveyed in [8].

In order to track routine location, Mason designed a system which allows participants to upload photos with GPS information [9]. In Noisemap, a smartphone was used as a noise meter to send noise information of the surroundings to a management platform [10]. [11] proposed an energy-efficient packet reporting scheme to report event packets in an energy-efficient manner. Amazon Mechanical Turk was a utility application for different tasks and requesters as a programmatic interface. A person or corporation acted as a platform who published tasks with a specified reward. Participants could complete these tasks, and then get some reward as incentives [12].

\subsection{Motivations and Related Works}

The architecture of a generic participatory sensing system is shown in Fig. 1, where there are two categories of main stakeholders, namely: platform and participants. When one type of sensory information is required, the platform publishes the task to suitable participants. Following some negotiation processes, the participants reach an agreement with the platform on their expected amount of rewards. Then, they collect sensory data and upload it to the platform. We focus on the negotiation processes and aim at designing an optimal incentive mechanism. On one hand, help platform collects enough data with affordable reward, on the other hand, help participants obtain more profit. 
The incentive mechanism is necessary for the participatory sensing system. Firstly, lots of costs may incur when taking part in a sensing task, such as network bandwidth usage, energy usage, time usage and so on. Thus, the rewards are necessary to encourage participants to take the task. Secondly, unlike traditional sensor networks, smart devices are rather personal, there is no sink node which control all devices' behaviors completely, only the owner of the device can decide when, where and how to use it for participation. Therefore, rewards should be paid to influence the decisions of participants, which aim to keep participatory sensing systems running and make sure of a satisfactory level of data quality and quantity.

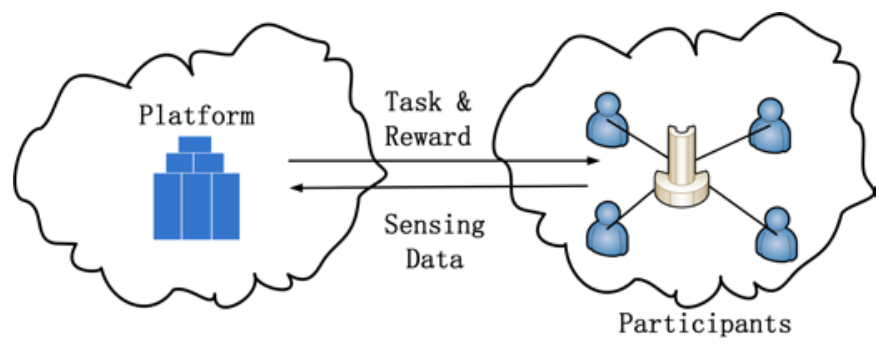

Fig. 1. The architecture of a participatory sensing system.

The incentive mechanisms have been widely implemented recently. Wang et al. modeled the dynamics of incentive mechanisms in autonomous networks [13]. Zhao et al. used the incentive protocol in peer-to-peer networks, and aimed at encouraging cooperation among end-nodes so as to deliver a scalable and robust service [14]. Huang et al. provided incentives for individual users of an ad-hoc network to cooperate with each other [15]. Song et al. proposed a data quality aware strategy with participants' reward [16]. The incentive mechanism was used in data quality aware systems to stimulate participants' involvement and enhance the system robustness [17]. Moreover, [18] investigate the frugal online incentive problem based on an online auction model.

There are two research challenges for participatory sensing system. The first one is how to recruit and retain more participants; the second one is how to improve the quality of data. As the key part of a participatory sensing system, the incentive mechanisms have been widely researched to solve the above problem. Lv et al. mainly focused on how to encourage existing participants to recruit more participants in [19]. Incentive mechanisms in [20-22] took trustworthiness of participants' data into consideration to improve the quality of it. [20] investigated community sensing, where sensors are controlled by self-interested agents that report their measurements to a center. [21] proposed a novel class of incentive protocols based on social norms which integrates reputation mechanisms into the existing pricing schemes currently implemented on crowdsourcing websites. In [22] Albers et al. studied coupons as incentives in order to stimulate users participation, especially in applications that rely on real-time data. The incentive strategy in [23-26] considered reputation to select trustworthy participants. In [23] a reputation system in which a reputation score is calculated for each device as a reflection of the trustworthiness of its sensor data is proposed, and the Gompertz function was used as a fundamental building block. [24] proposesd a new class of incentive mechanisms aiming at compelling self-interested users in online communities to cooperate with each other by exchanging resources or services. In [25] two privacy-aware incentive schemes for mobile sensing to promote user participation were given. [26] proposed a framework called ARTSense to solve the problem of "trust without identity" in participatory sensing networks. In order to use incentive mechanisms to attract more participants, Cheng et 
al. widened the scope of incentives from device/user level to the group level [27]. Zhang et al. in [28] and Zhao et al. [29] took fairness problem into consideration and proposed its own activities solutions. In [29-32] the cooperative game theory has also been used to design the incentive mechanisms. [30] proposed a game-theoretic framework to model user behavior and designed incentive-based strategies to stimulate user cooperation in peer-to-peer live streaming. In [31], the problem of grid-to-vehicle energy exchange between a smart grid and plug-in electric vehicle groups (PEVGs) is studied using a noncooperative Stackelberg game. The authors in [32] proposed decentralized solutions for resource negotiation, where multiple autonomous users self-organize into a coalition which shares the same network resources and negotiate the division of these resources by exchanging information about their requirements. The incentive strategy in [33] took participant's privacy into consideration.

The privacy and reputation are equally important and have a significant impact on the incentive mechanism. However, they have been considered separately in the prior works. And if the incentive mechanism only takes part of the factors into consideration, a potential risk may arise and the quality of data can not be guaranteed effectively. Motivated by the reason above, we study an optimal incentive mechanism based on cooperative game theory, which takes the participants' privacy and reputation into consideration jointly. With the proposed incentive mechanism, the total amount of sensory data can be maximized, and the quality of the data will be improved.

The difference between the mechanism proposed in this work and our previous work [34] is focused on two aspects: firstly, the mechanism in [34] only takes the participants' reputation into consideration, while the mechanism in this work considers both privacy and reputation jointly; Secondly, the mechanism in [34] just involves one incentive algorithm, while the mechanism in this work proposes two algorithms, one for reach the Stackelberg equilibrium, and the other show how to employ the incentive strategy for the platform.

\subsection{Main Contributions}

The main contributions of this paper can be summarized as follows:

- We designed an incentive mechanism in participatory sensing for ambient assisted living, which is the first time that the participants' privacy and reputation are taken into consideration jointly.

- We formulated a Stackelberg game mode and discussed the properties of the game, and we proved that there exists a unique Stackelberg equilibrium. Two algorithms are proposed in this paper, one for reach the Stackelberg equilibrium, and the other show how to employ the incentive strategy for the platform.

- Finally, extensive simulations have validated the effectiveness of the proposed online incentive mechanism. With the mechanism, the total amount of sensory data can be maximized and the quality of the data can be guaranteed effectively.

\subsection{Paper Outline}

The rest of this paper is organized as follows. In Section 2, we discuss the key elements of our incentive strategy and the appropriate procedures to employ it. In Section 3, we analyze the profit of participant and platform. After that, we analyze the constraints for both participant and platform and propose a formulation model in Section 4. In Section 5, a Stackelberg game model is formulated and discussed, and an efficient algorithm is proposed to reach Stackelberg equilibrium. In order to evaluate the performance of our incentive strategy, simulations are done in MATLAB and the results are shown in Section 6. Section 7 concludes the paper. 


\section{The Design of the Incentive Strategy}

We aim at designing an incentive strategy that can recruit more participants and promote them to try their best. Virtual currency is applied in our mechanism to motivate participants, which is a kind of currency used on the internet and will be paid to participants who take the task as a reward.

There are some usages of reputation in incentive strategy [23-25]. The main functionality of reputation is to avoid malicious participants as much as possible. Comparing with rewards, which represent and characterize the transient quality of the task to be done, a participant's reputation is rather a long-term, accumulated metric to identify the quality and trustworthiness of a participant's credibility.

To improve the quality of the task we consider the reputation mechanism, which influences the reward. If participants want to have a good reputation, they will try their best to improve the quality of the work. The idea is: when data is uploaded, the platform updates participant's reputation according to the quality of the data, and pays the participant according to its reputation and the data.Which means the more effort the participant has made, the higher reputation score and the more reputation reward it will get.

The procedures of employing the incentive strategy are shown in Fig. 2. Firstly, the platform requests the nearby devices to announce their states, such as remaining power, the free storage space, the opportunity consumption, etc.. Secondly, the platform checks their states, which have been received from the participants, and makes the incentive strategy. Thirdly, the platform transmits the incentive mechanism to devices with the announcement of the reward for the task. If an agreement can be reached, the negotiation is accomplishedsuccessfully. Fourthly, the participants take the sensing task and transmit data to the platform.Finally, the platform evaluates the quality of data, updates the participant's reputation and pays the reward to the participant according to the quality of data and participant's reputation.

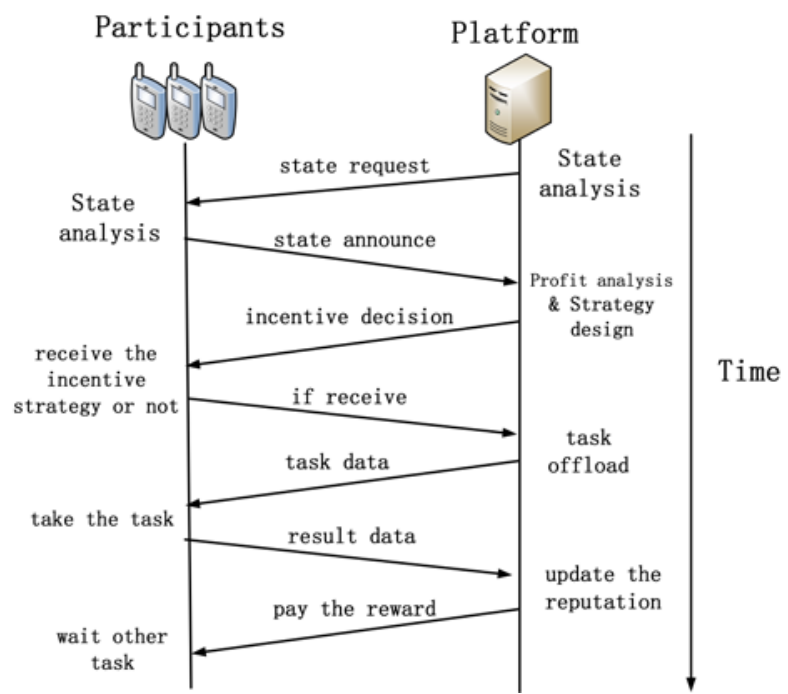

Fig. 2. The procedures of employing the incentive strategy. 


\section{The System Model and the Analysis of Profit}

\subsection{The System Model}

Let $\mathrm{D}=\left\{d_{1}, d_{2}, \cdots, d_{n}\right\}$ be the set of smart devices, $d_{i}(1 \leq i \leq n)$ is one smart device of the set. The initial power and remaining power of $d_{i}$ can be denoted as $\mathrm{e}_{i}^{0}, \mathrm{e}_{i}$ respectively. The used storage space and the initial free storage space of $d_{i}$ can be written as $m_{i}, m_{i}^{0}$ separately. The states of device $d_{i}$ can be described as $s_{i}=\left(e_{i}, m_{i}, e_{i}^{0}, m_{i}^{0}\right)$. Since the device has limited energy and storage space, when a participant has no energy or no free storage space it can do nothing anymore. Therefore, we give the definition of participant's opportunity consumption as follow.

Definition 1. The participant's opportunity consumption is defined as:

$$
h\left(e_{i}, m_{i}, e_{i}^{0}, m_{i}^{0}\right)=\frac{e_{i}^{0}}{e_{i}}+\frac{m_{i}^{0}}{m_{i}^{0}-m_{i}}
$$

The map and contour map of participant's opportunity consumption are shown in Fig. 3 and Fig. 4. Where $e_{i} / e_{i}^{0}$ denotes the proportion of remaining/ initial power power, and $m_{i} / m_{i}^{0}$ denotes the proportion of the used/ initial free storage space and the . The less the remaining power and the free storage space is, the more opportunity consumption will be.

In our model, an opportunity consumption threshold is introduced. When the participant's opportunity consumption is larger than the threshold, it will not take part in the sensing task. Otherwise, it will take an appropriate action according to the profit. This method aims at avoiding consumption when the participant has a low remaining energy level or a low free storage space level.

In our model, we limit $h\left(e_{i}, m_{i}, e_{i}^{0}, m_{i}^{0}\right) \leq h_{0}$ and set $h_{0}=20$ in practical application. The safety range of device's state is shown in Fig. 4. The device whose state is not in the safety range will not take part in the sensing task. Meanwhile, the device whose state is in the safety range will consider whether to take the task based on its profit.

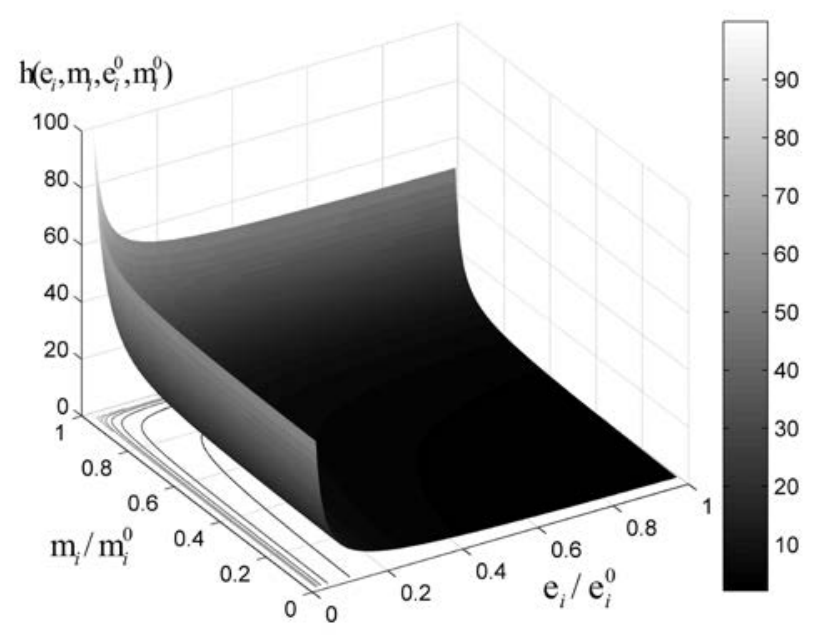

Fig. 3. Participant's opportunity consumption. 


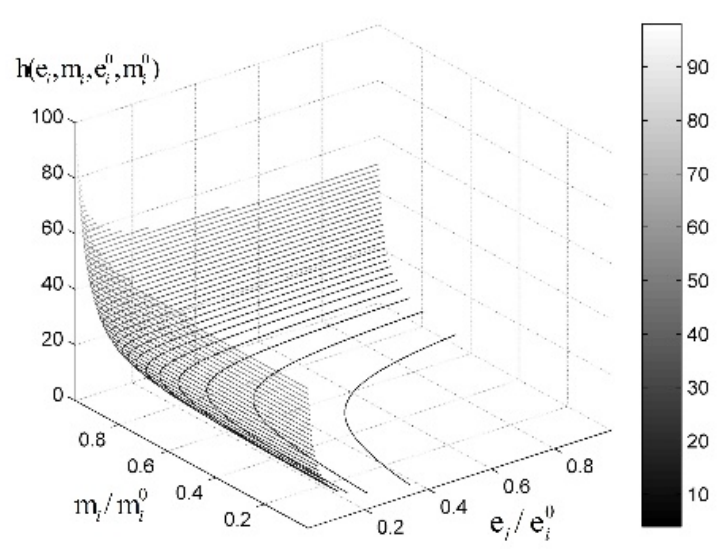

Fig. 4. The contour map of participant’s opportunity consumption.

\subsection{The Analysis of Profit}

In the progress of participatory sensing, the participant can get a certain amount of reward but cost energy and might leak their privacy. The platform can get the utility of information but it must pay for it. Our incentive strategy focuses on the profits of the participants and platform, assuming that the participants and the platform have the tendency towards the profit and their principles are profits maximization.

When participant $d_{i}$ uploads data to the platform, the utility of the data will be formulated as

$$
O_{i}=f\left(a_{i}, b_{i}\right)+\theta_{i}
$$

Where $a_{i}$ denotes the effort of $d_{i}$ has made, and $b_{i}$ denotes the reputation of $d_{i}, f\left(a_{i}, b_{i}\right)$ is one part of utility related with $a_{i}$ and $b_{i}, \theta_{i} \sim N\left(0, \sigma_{1}^{2}\right)$ denotes the other part of utility related with uncertainty factors.

The participant devices must pay for the sensing process, the costs may be the free storage space, the energy, the time and the attention. In our work, we formulate the basic consumption of effort $a_{i}$ as $g\left(\mathrm{a}_{i}\right)$.When participants uploading their sensory data to the platform, the gathered sensory readings may reveal sensitive information of the participants to others [33]. Our incentive mechanism takes the private of participants into consideration, and takes the leak of privacy as one kind of consumption. We define the leak of privacy as $p r$.Sum up the above analysis, the function to compute the total costs of participant $d_{i}$ as:

$$
S_{i}=g\left(a_{i}\right)+h\left(e_{i}, m_{i}, e_{i}^{0}, m_{i}^{0}\right)+p r_{i}
$$

A good incentive mechanism is inherently related to recruit enough participants and encourage participants to contribute more quality data. Every participate in the sensing progress will get a fixed rewards $\alpha$ regardless of the quality of the data. To encourage participants provide more quality data, extra rewards will be given to the participant that makes more contribution, so we introduce the incentive intensity factor $\beta$. In the participatory 
sensing progress, the reward $G_{i}$ will be given to the participant $d_{i}$, we define $G_{i}$ as:

$$
G_{i}=\alpha+\beta O_{i}
$$

Where $\alpha$ is the fixed reward for the participant, and $\beta$ is an incentive intensity factor for the utility of the data.

The pure profit of participant $d_{i}$ in the whole participatory sensing progress is given as follow:

$$
P G_{i}=G_{i}-S_{i}=\alpha+\beta f\left(\mathrm{a}_{i}, \mathrm{t}_{i}\right)-\mathrm{g}\left(\mathrm{a}_{i}\right)-\mathrm{h}\left(\mathrm{e}_{i}, \mathrm{~m}_{i}, \mathrm{e}_{i}^{0}, \mathrm{~m}_{i}^{0}\right)-p r_{i}
$$

In this incentive mechanism, the more efforts, the more participant rewards it will get, of course its cost will be higher. That means, in the participatory sensing progress the cost is closely related to the reward, the pure profit is determined by the value of reward and cost.

The profit of platform $u$ is:

$$
G_{u}=f\left(a_{i}, b_{i}\right)+E(\theta)
$$

As $\theta_{i} \sim N\left(0, \sigma_{1}^{2}\right)$, so $E(\theta)=0$. The reward that the platform should pay for is:

$$
P_{u}=\alpha+\beta f\left(a_{i}, b_{i}\right)
$$

Therefore, the pure profit of platform $u$ is:

$$
P G_{u}=G_{u}-P_{u}=f\left(a_{i}, b_{i}\right)-\left[\alpha+\beta f\left(a_{i}, b_{i}\right)\right]
$$

\section{The Problem Formulation}

\subsection{The Constraint of Effort \& Rewards}

When a certain type of sensory data is required, the platform publishes a corresponding sensing task with the detailed quality of data, together with the amount of affordable rewards that will be paid to the participants. As the more effort the participant makes, the better quality sensory data will be. There are two constraints, one is for the effort: $a_{i} \geq A$, where $A$ is the threshold of the effort that the participant should make; the other is the reward should be affordable: $\alpha \leq \alpha_{0}, \beta \leq \beta_{0}$. Where $\alpha_{0}$ and $\beta_{0}$ are the threshold of the rewards.

\subsection{The Constraint of Opportunity Consumption}

As discussed in section 3.1, the smart device has limited energy and storage space, when a participant has no energy or no free storage space he can do nothing anymore. The device $d_{i}$ who wants to participate in the sensing task should stay in the feasible state range, namely $h\left(e_{i}, m_{i}, e_{i}^{0}, m_{i}^{0}\right) \leq h_{0}$.

\subsection{The Constraint of Participation}

Participant can do something else if it does not take part in this sensing task, and gets profit in other ways. Therefore, the profit of participant $P_{i}$ should be more than its opportunistic profit, which is.

$$
\alpha+\beta f\left(a_{i}, b_{i}\right)-\mathrm{g}\left(a_{i}\right)-\mathrm{h}\left(\mathrm{e}_{i}, \mathrm{~m}_{i}, \mathrm{e}_{i}^{0}, \mathrm{~m}_{i}^{0}\right)-p r_{i} \geq \bar{U}
$$

Where $\bar{U}$ denotes the opportunistic profit of participant if he does not take part in the sensing task. 


\subsection{The Constraint of Maximal Profit}

In the perspective of the platform, the total pure profit must be positive: $P G_{i} \geq 0$. Then the incentive mechanism should be designed to maximize its pure profit $P G_{u}$. This can be expressed as:

$$
\max \left\{f\left(a_{i}, b_{i}\right)-\left[\alpha+\beta f\left(a_{i}, b_{i}\right)\right]\right\}
$$

\subsection{The Formulation of the Model}

above all, in the participatory sensing progress the optimal incentive model for the platform can be formulated as:

$$
\left.\begin{array}{c}
\max \left\{f\left(a_{i}, b_{i}\right)-\left[\alpha+\beta f\left(a_{i}, b_{i}\right)\right]\right\} \\
a_{i} \geq A(1 \leq i \leq n), \alpha \leq \alpha_{0}, \beta \leq \beta_{0} \\
f\left(a_{i}, b_{i}\right)-\left[\alpha+\beta f\left(a_{i}, b_{i}\right)\right] \geq 0
\end{array}\right\}
$$

The optimal incentive model for participant $d_{i}$ can be formulated as:

$$
\left.\begin{array}{c}
\max \left[\alpha+\beta f\left(a_{i}, b_{i}\right)-\mathrm{g}\left(a_{i}\right)-h\left(e_{i}, m_{i}, e_{i}^{0}, m_{i}^{0}\right)-p r_{i}\right] \\
\mathrm{h}\left(e_{i}, m_{i}, e_{i}^{0}, m_{i}^{0}\right) \leq h_{0}\left(h_{0}=20\right) \\
\alpha+\beta f\left(a_{i}, b_{i}\right)-\mathrm{g}\left(a_{i}\right)-\mathrm{h}\left(\mathrm{e}_{i}, \mathrm{~m}_{i}, \mathrm{e}_{i}^{0}, \mathrm{~m}_{i}^{0}\right)-p r_{i} \geq \bar{U}
\end{array}\right\}
$$

The solution $\left(\beta^{*}, a_{i}^{*}, \alpha^{*}\right)$ satisfies models (11) and (12) is the optimal strategy for participant $d_{i}$ and platform $u$ within the participatory sensing progress.

In our model, we define the output ofparticipant $d_{i}$ as:

$$
f\left(a_{i}, b_{i}\right)=k_{1} a_{i}+k_{2} b_{i}
$$

As the reputation of participant closely relates to the quality of the data, we define the reputation as:

$$
b_{i}=b_{i 0}+\lambda a_{i}
$$

Where $b_{i 0}$ denotes the initial reputation of participant $d_{i}, \lambda$ is a constant which reflects the relationship between participant's effort and reputation.

The basic consumption for effort $a_{i}$ is:

$$
g\left(a_{i u}\right)=k_{3} a_{i}^{2}
$$

As $\alpha$ is a constant which regardless of the quality of the sensory data, we define it as:

$$
\alpha^{*}=\frac{1}{2 m} \sum_{i=1}^{m}\left[h\left(e_{i}, m_{i}, e_{i}^{0}, m_{i}^{0}\right)+p r_{i}\right]
$$

Where $m$ is the number of devices that have announced their states. 
$\frac{1}{m} \sum_{i=1}^{m}\left[h\left(e_{i}, m_{i}, e_{i}^{0}, m_{i}^{0}\right)+p r_{i}\right]$ is the mean of those participants' opportunity consumption and privacy consumption, and $\alpha^{*}$ is a half of the mean.

Take formulas (13), (14) and (15) into equation (5) we obtain:

$$
P G_{i}=\alpha^{*}+\beta\left[k_{1} a_{i}+k_{2}\left(b_{i 0}+\lambda a_{i}\right)\right]-\mathrm{k}_{3} a_{i}^{2}-\left(\frac{e_{i}^{0}}{e_{i}}+\frac{m_{i}^{0}}{\mathrm{~m}_{i}^{0}-\mathrm{m}_{i}}\right)
$$

\section{The Approach of the Stackberg Game}

\subsection{The Design of the Stackelberg Game}

The Stackelberg game [31] which known as a leader-follower game, studies the decision making processes of a number of independent players.

As discussed in section 2, within the participatory sensing progress, the participants determine the amount and the quality of the sensory data. Based on the incentive strategy made by the platform, we model our problem as a Stackelberg game.

$$
\Gamma=\left\{\left(\left\{\mathrm{d}_{i}\right\}_{1 \leq i \leq n}, u\right),\left\{a_{i}\right\}_{1 \leq i \leq n},\left\{P G_{i}\right\}_{1 \leq i \leq n},(\alpha, \beta), P G_{u}\right\}
$$

In the above formulation, $\left(\left\{\mathrm{d}_{i}\right\}_{1 \leq i \leq n}, u\right)$ is the set of players within the game, where $u$ is the platform who is the leader and the $i$-th participant $d_{i}$ is the follower. $a_{i}$ is the effort strategy of follower $d_{i}$, and $P G_{i}$ is the pure profit of follower $d_{i}$ by choosing the strategy $a_{i} \cdot P G_{u}$ is the pure profit of platform with incentive strategy $(\alpha, \beta)$. After the game, platform $u$ determines the reward, which will be paid for the participants, and participant $d_{i}$ decides the amount of data it will upload.

As the leader, the platform chooses its strategy $(\alpha, \beta)$ in the first step, while device $d_{i}$ independently decides $a_{i}$ with $(\alpha, \beta)$ in the second step. Considering the Stackelberg game $\Gamma$, a set of strategies $\left(\alpha^{*}, \beta^{*}, a_{i}^{*}\right)$ reaches the Stackelberg equilibrium if it satisfies:

$$
\begin{gathered}
P G_{i}\left(a^{*}, \alpha^{*}, \beta^{*}\right) \geq P G_{i}\left(a^{* *}, \alpha^{*}, \beta^{*}\right), \forall i \\
P G_{u}\left(a^{*}, \alpha^{*}, \beta^{*}\right) \geq P G_{u}\left(a^{*}, \alpha^{*}, \beta^{* *}\right)
\end{gathered}
$$

\subsection{The Analysis of Equilibrium}

First of all, we discuss the Stackelberg equilibrium of the game.

Lemma 1. There exists a unique Stackelberg equilibrium for the proposed Stackelberg game $\Gamma$.

Proof: Firstly, we show the existence of Stackelberg equilibrium for the seller. In the second step of the game, the follower device $d_{i}$ decides the effort it wants to provide, so as to maximize $P G_{i}$. We can easily check that $P G_{i}$ is a strictly concave function of $a_{i}$, as the second derivation $P G_{a_{i}}^{\prime \prime}=-2 k_{3}<0$ where $k_{3}>0$. Thus, the decision made by each participant has a unique solution, with an offered incentive strategy $(\alpha, \beta)$. Secondly, we check the existence of 
Stackelberg equilibrium for the platform. Since the platform is aware of the decisions made by the participants in the second stage, we first discuss the decision made by each participant. As discussed above, with a given incentive strategy $(\alpha, \beta)$, participant $d_{i}$ determines $a_{i}$ based on the following equation:

$\frac{\partial P G_{i}}{\partial a_{i}}=\frac{\partial\left\{\alpha+\beta\left[k_{1} a_{i}+k_{2}\left(b_{i 0}+\lambda a_{i}\right)\right]-\mathrm{k}_{3} a_{i}^{2}-\left(\frac{e_{i}^{0}}{e_{i}}+\frac{m_{i}^{0}}{\mathrm{~m}_{i}^{0}-\mathrm{m}_{i}}\right)\right\}}{\partial a_{i}}=0$. We can get: $a_{i}=\beta\left(k_{1}+\lambda k_{2}\right) / 2 k_{3}$,

since $a_{i} \geq A$, the best choice made by $d_{i}$ is as follow:

$$
a_{i}^{*}=\max \left[\beta\left(k_{1}+\lambda k_{2}\right) / 2 k_{3}, A\right]
$$

With the information of $a_{i}^{*}$, we can rewrite $P G_{u}$ into:

$$
P G_{u}=-\left(k_{1}+\lambda k_{3}\right)^{2} \beta^{2} / 2 k_{3}+\left[\left(k_{1}+\lambda k_{3}\right)^{2} \beta^{2} / 2 k_{3}-k_{2} b_{i 0}\right] \beta+k_{2} b_{i 0}-\alpha
$$

According to Eq. (21), we get the derivation $P G_{u \beta}{ }^{\prime \prime}=-\left(k_{1}+\lambda k_{3}\right)^{2} / k_{3} \leq 0$, hence $P G_{u}$ is a strictly concave function of $\beta$ in response to the decisions made by participant. Thus, there exists a unique Stackelberg equilibrium for $\Gamma$.

\subsection{Algorithm to Reach Stackelberg Equilibrium}

In this section, we show the detail of how the platform and the participants make their decisions to maximize their own profits. We propose a ternary search based algorithm. As discussed above, we can get $\alpha^{*}$ as formula (16). To get $\beta^{*}$, specifically we set four points between 0 and $\beta_{0}$, denoted as $L$, mid $_{1}$, mid $_{2}, R$. Initially, we

set $L=0, R=\beta_{\max }, \operatorname{mid}_{1}=L+(R-L) / 3, \operatorname{mid}_{2}=L+2(R-L) / 3$. The progress is conducted as follows:

1) With the incentive mechanism $\left(\alpha^{*}, \operatorname{mid}_{1}\right)$, we can calculate the optimal decision $a_{i}^{*}$, made by each participant according to model (20). Then, according to $a_{i}^{*}$ and $\left(\alpha^{*}, \operatorname{mid}_{1}\right)$, we get the pure profit $P G_{u}$,we denote it as $P G_{u}{ }^{\text {mid1 } 1}$.

2) Similarly, with incentive strategy $\left(\alpha^{*}, \operatorname{mid}_{2}\right)$, we calculate $a_{i}^{*}$ and the value of $P G_{u}$, which is denoted as $P G_{u}^{\text {mid } 2}$.

3) Then we compare $P G_{u}{ }^{\text {mid } 1}$ and $P G_{u}^{\text {mid } 2}$. If $P G_{u}{ }^{\text {mid } 1}<P G_{u}^{\text {mid } 2}$, the maximum value of $P G_{u}$ must be located between $\operatorname{mid}_{1}$ and $R$ because of the concave of $P G_{u}$, so we set $L=$ mid $_{1}$, otherwise we set $R=\operatorname{mid}_{2}$.

We continue the above process until the difference between $\mathrm{L}$ and $\mathrm{R}$ is less than the minimal unit of the effort. The detailed process is presented in Algorithm 1, where $\varepsilon$ is supposed to be the minimal unit of the effort. 


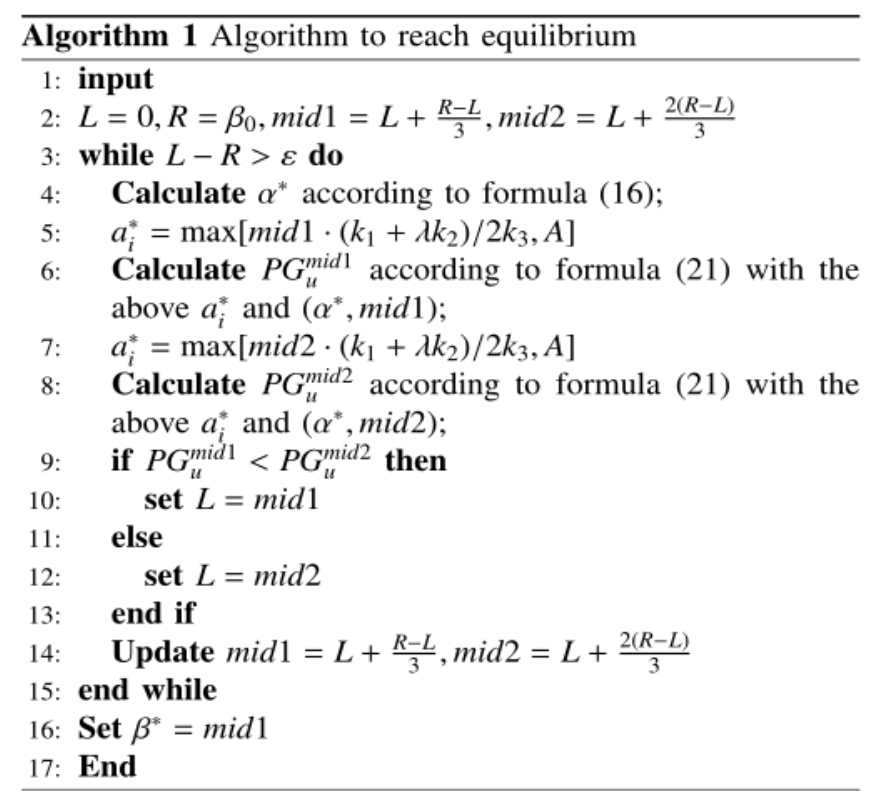

Theorem 1. The proposed Algorithm 1 can reach the Stackelberg equilibrium of the game. Proof: Due to the strict concave of $P G_{u}$, the ternarysearch used in Algorithm 1 must be able to find the decision of $\left(\alpha^{*}, \beta^{*}\right)$ that maximize the value of $P G_{u}$. In response to $\left(\alpha^{*}, \beta^{*}\right)$, each participant $d_{i}$ decides its effort $a_{i}$ to maximize its utility function $P G_{i}$. Therefore, participant $d_{i}$ also reaches a fixed point at which its profit is maximized with effort $a_{i}^{*}$. Hence, the proposed algorithm can reach the Stackelberg equilibrium of the game.

If those constraints in model (11) and (12) can be satisfied completely, the participatory sensing work can be done successfully. Then the reward will be paid for the participant is:

$$
G_{i}=\alpha^{*}+\beta^{*}\left[k_{1} a_{i u}^{*}+k_{2}\left(b_{i 0}+\lambda a_{i u}^{*}\right)\right]
$$

Then the pure profit of participant is:

$$
P G_{i}=\alpha^{*}+\beta^{*}\left[k_{1} a_{i u}^{*}+k_{2}\left(b_{i 0}+\lambda a_{i u}^{*}\right)\right]-k_{3} a_{i u}^{* 2}-\mathrm{h}\left(\mathrm{e}_{i}, \mathrm{~m}_{i}, \mathrm{e}_{i}^{0}, \mathrm{~m}_{i}^{0}\right)
$$

The pure profit of task platform $d_{u}$ is:

$$
P G_{u}=k_{1} a_{i}^{*}+k_{2}\left(b_{i 0}+\lambda a_{i}^{*}\right)-\alpha^{*}-\beta^{*}\left[k_{1} a_{i}^{*}+k_{2}\left(b_{i 0}+\lambda a_{i}^{*}\right)\right]
$$

\subsection{Algorithm to Employ the Incentive Strategy}

As discussed in section 2, the method to employ the incentive strategy for the platform is shown in Algorithm 2.

1) The platform requests the nearby devices announce their states, such as remaining power, free storage space, opportunity consumption, etc.

2) The platform checks the states of these devices that received from the participants and makes the incentive strategy $\left(\alpha^{*}, \beta^{*}\right)$ according to Algorithm 1.

3) The platform transmits the incentive mechanism to devices that announces the reward for the task. If the devices reach an agreement with the platform, they will take the sensing task and transmit the data to the platform. 
4) The platform evaluates the quality of the data and updates the participant's reputation, then pay a reward to the participant according to the quality of data and participant's reputation.

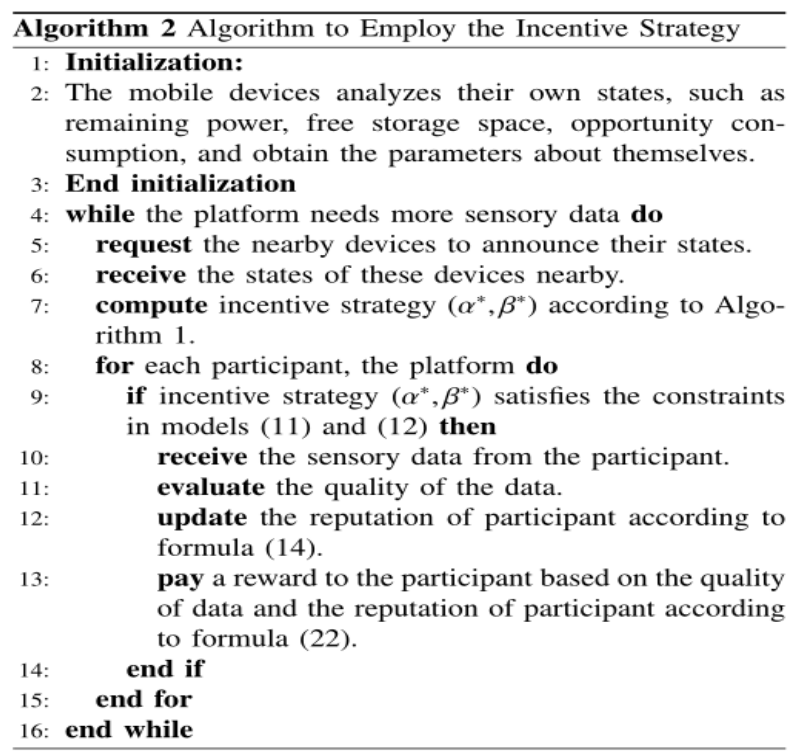

\subsection{The Analysis of Algorithm Complexity}

Within Algorithm 1, a "While" loop is used and the number of cycles is constant. Therefore, the computational complexity of Algorithm 1 is $o(1)$. Algorithm 2 consists of a "While" loop that embeds a "For" loop. The numbers of cycles of the two loops are both depend on $n$, which is the number of nodes. The number of cycles of the "While" loop is less than $n$, and $n$ is the upper bound of it. So, the computational complexity of Algorithm 2 is no more than $o\left(n^{2}\right)$. When the value of $n$ is not too large, the computational complexity of Algorithm 2 is achievable. In one word, the computational complexity of the algorithms proposed in this paper is affordable.

\section{Experimental Evaluation}

This section presents simulation-based evaluation of the proposed incentive mechanism. The simulation setup is outlined in Section 6.1 and the results are shown in Section 6.2.

\subsection{Simulation Setup}

To undertake the preliminary evaluations outlined herein, we chose to conduct simulations, as real participatory sensing experiments in social are difficult to organize. Simulations afford a controlled environment where we can carefully vary certain parameters and observe the impact on the system performance. We choose MATLAB simulator for the purpose.

In order to investigate the performance of the proposed incentive mechanism and obtain reliable results, the scene is designed in Fig. 6. We assumed that 20 nodes are distributed randomly within an area of $200 \mathrm{~m} * 200 \mathrm{~m}$, where the mobility model of each node was random waypoint model with the speed of $10 \mathrm{~m} / \mathrm{s}$. The participatory sensing task is collecting the 
circumstance information of the sensitive areas once a second. These sensitive areas are uniform distribution, and every sensitive area is a circular with $\mathrm{r}=30 \mathrm{~m}$. We do not care about how the circumstance information is acquired, and assume everyone in the sensitive areas can sense the information.

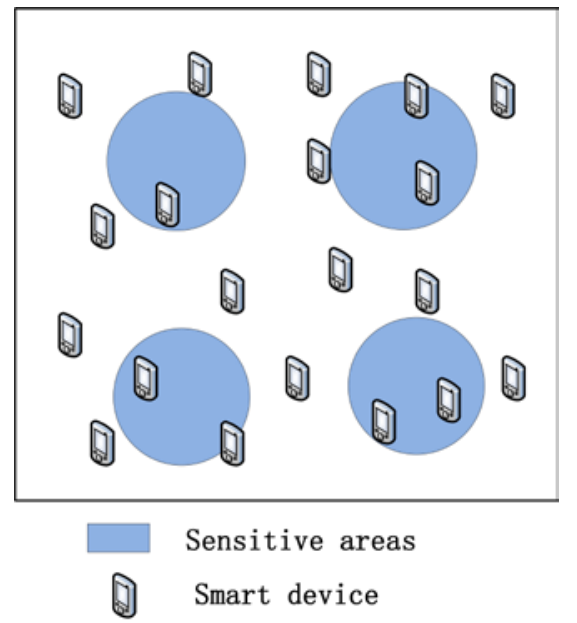

Fig. 6. The scene of simulation.

In the simulation, the various parameters are set as follows, $k_{1}=5, k_{2}=2, k_{3}=3$, $\sigma^{2}=8, \bar{U}=10, \lambda=0.5, b_{i 0}=1, h_{0}=20$, then all the participants' initial power $e_{i}^{0}$ and initial storage space $m_{i}^{0}$ are all 10, participants' remaining power is uniformly distributed $e_{i} \sim U(0,10)$ and the free storage space is uniformly distributed $m_{i} \sim U(0,10)$, the privacy consumptions of participants are exponential distribution $\operatorname{pr}_{i} \sim N(3,5)$, the threshold of the rewards for the platform is $\alpha_{0}=15$ and $\beta_{0}=5$. The results are shown in Section 6.2.

\subsection{The Results of Simulation}

Fig. 7 depicts the value of the fixed rewards the platform affords for participants with time going on. As shown in the figure, the platform changes its fixed rewards in the beginning and stays stable gradually. It is reasonable, as the fixed reward is related to the mean of participants' opportunity consumption and privacy consumption. At the beginning, there are few participants and the mean changes a lot, as there are more and more participants with time going on, the mean remains stable relatively. 


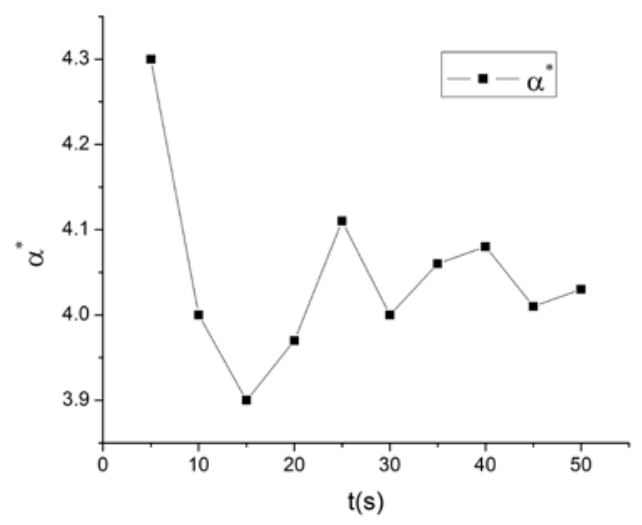

Fig. 7. The value of fixed rewards.

Fig. 8 depicts the number of smart device in the sensitive area and the number of participants among those devices. We can see that not all devices in the sensitive area prefer to take part in the sensing task, as there are some constraints for the participants, such as opportunity consumption constraint and participation constraint. At the same time, the platform can not pay unlimited reward as the reward constraint. However, almost all devices in the sensitive area take part in the sensing task, that means our incentive strategy performs well.

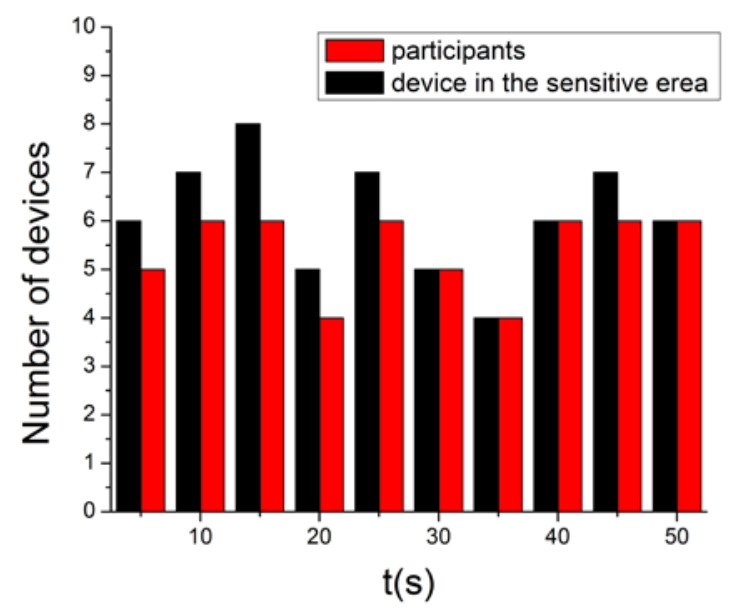

Fig. 8. The number of participant and device within the sensitive area.

Fig. 9 shows the award for participant and the quantity of data they uploaded at that time. As there are fewer participants from 25 seconds to 35 seconds, the platform collects fewer amount of data, and pays less reward at the same time. As shown in the figure, the quantity of data and the reward has a positive relationship, that is to say, to collect a larger amount of data, the platform needs to pay more award for the participants. 


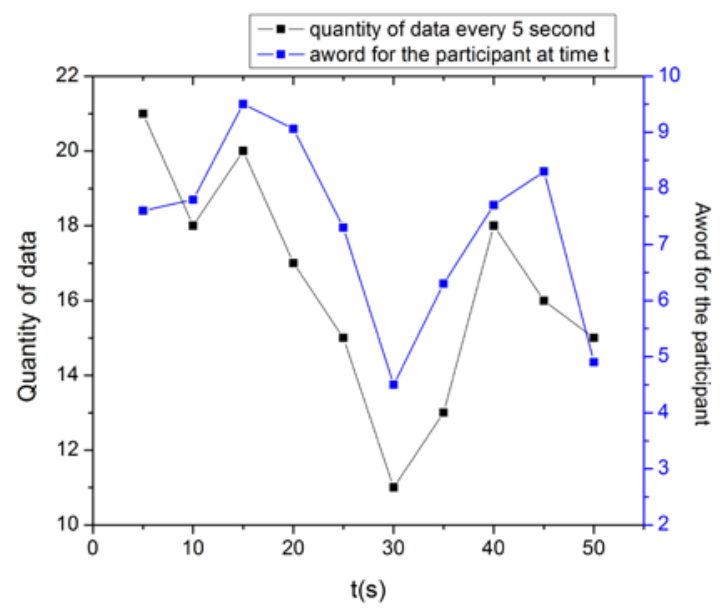

Fig. 9. Quantity of data and award for participant.

Fig. 10 shows the total pure profit of the platform and the total pure profit of participants.It is visiable that, both the pure profit of the platform and participants are all increase steadily with time going on. The reason is that with our incentive mechanism the platform can recruit enough participants, encourage participants to contribute high quality data and get the utility of the data. At the same time, these participants can get enough rewards to offset their costs.

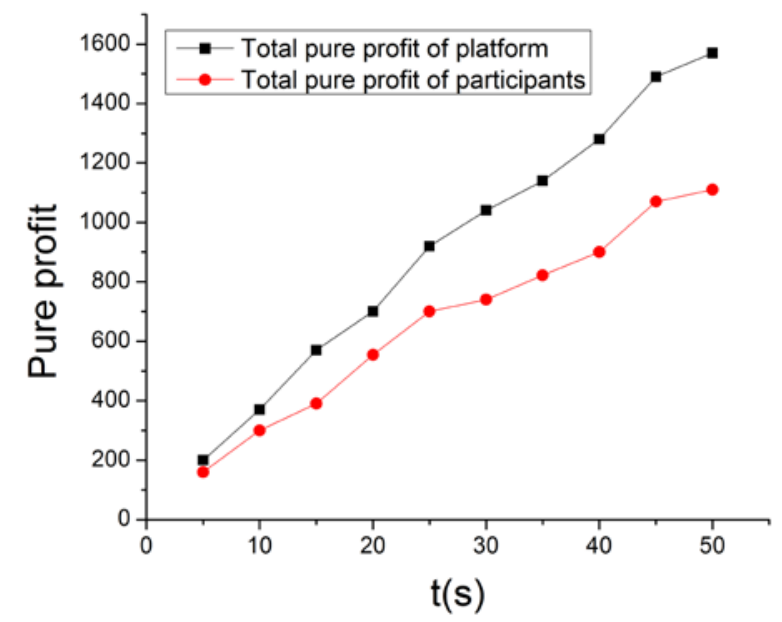

Fig. 10. The total pure profit of platform and participants.

\section{Conclusions}

Ambient assisted living is an intelligent system that enables a healthier and safer life in the preferred living environment. Participatory sensing has shown its great potential in data acquisition for ambient assisted living. In this paper, we proposed an incentive mechanism for ambient assisted living in data acquisition with participatory sensing. Firstly, we discussed the key elements of the incentive strategy and the appropriate procedures to employ an incentive strategy. Secondly, we analyse the profits of the task platform and participants, in the progress, 
we take the state and reputation of participant into consideration. Thirdly, a Stackelberg game model is formulated and the property of the game is discussed, then we proved that there exists a unique Stackelberg equilibrium. Furthermore, an efficient algorithm is given to reach the Stackelberg equilibrium, and the other is given to show how to employ the incentive strategy for the platform. Finally, we conducted the simulation to evaluate the properties and effectiveness of the proposed mechanism; the results show that with our incentive mechanism the performance of the system will be improved.

\section{References}

[1] D. Cook, S. Das, "Smart Environments: Technologies, Protocols and Applications," Wiley-Interscience, pp. 192 - 197, 2005. Article (CrossRef Link)

[2] S. Poslad, "Ubiquitous Computing: Smart Devices, Environments and Interactions," Wiley, Mar. pp. 540-545, 2009. Article (CrossRef Link)

[3] H. Gao et al., "A Survey of Incentive Mechanisms for Participatory Sensing," in IEEE Communications Surveys \& Tutorials, vol. 17, no. 2, pp. 918-943 Secondquarter 2015.

Article (CrossRef Link)

[4] N. D. Lane, E. Miluzzo, H. Lu, D. Peebles, T. Choudhury and A. T. Campbell, "A survey of mobile phone sensing," IEEE Communications Magazine, vol. 48, no. 9, pp. 140-150, Sep. 2010. Article (CrossRef Link)

[5] S. S. Kanhere, "Participatory Sensing: Crowdsourcing Data from Mobile Smartphones in Urban Spaces," in Proc. of 2011 IEEE 12th International Conference on Mobile Data Management, Lulea, pp. 3-6, 2011. Article (CrossRef Link)

[6] N. Haderer, R. Rouvoy and L. Seinturier, "A preliminary investigation of user incentives to leverage crowdsensing activities," in Proc. of 2013 IEEE International Conference on Pervasive Computing and Communications Workshops (PERCOM Workshops), San Diego, CA, pp. 199-204, 2013. Article (CrossRef Link)

[7] I. Bisio et al.,"A Smartphone-Centric Platform for Remote Health Monitoring of Heart Failure," Wiley International Journal of Communication Systems, Article first published online: 14 Apr. 2014. Article (CrossRef Link)

[8] Z. Xiao, H. B. Lim and L. Ponnambalam, "Participatory Sensing for Smart Cities: A Case Study on Transport Trip Quality Measurement," IEEE Transactions on Industrial Informatics, vol. 13, no. 2, pp. 759-770, April 2017. Article (CrossRef Link)

[9] A. D. Mason, G. Michalakidis and P. J. Krause, "Tiger Nation: Empowering citizen scientists," in Proc.2012 6th IEEE International Conference on Digital Ecosystems and Technologies (DEST), Campione d'Italia, pp. 1-5, 2012. Article (CrossRef Link)

[10] I. Schweizer, C. Meurisch, J. Gedeon, R. B"artl, and M. M"uhlh"auser, "Noisemap: Multi-tier incentive mechanisms for participative urban sensing," in Proc. of the Third International Workshop on Sensing Applications on Mobile Phones, PhoneSense '12, ACM, New York, NY, USA, pp. 91-95, Nov.2012. Article (CrossRef Link)

[11] G. Abdul-Salaam, A. Hanan Abdullah and M. Hossein Anisi, "Energy-Efficient Data Reporting for Navigation in Position-Free Hybrid Wireless Sensor Networks," IEEE Sensors Journal, vol. 17, no. 7, pp. 2289-2297, April 1, 2017. Article (CrossRef Link)

[12] W. Mason, and D. J. Watts, "Financial incentives and the "performance of crowds," in Proc. of the ACM SIGKDD Workshop on Human Computation, HCOMP '09, ACM, New York, NY, USA, pp. 77-85, 2009. Article (CrossRef Link)

[13] Y. Wang, A. Nakao, and A. V. Vasilakos, "Heterogeneity playing key role: Modeling and analyzing the dynamics of incentive mechanisms in autonomous networks, " ACM Transactions on Autonomous and Adaptive Systems (TAAS), Vol. 7, No. 3, Sep. 2012, Article No. 31. Article (CrossRef Link) 
[14] B. Q. Zhao, J. C. S. Lui and D. M. Chiu, "A Mathematical Framework for Analyzing Adaptive Incentive Protocols in P2P Networks," IEEE/ACM Transactions on Networking, vol. 20, no. 2, pp. 367-380, Apr. 2012. Article (CrossRef Link)

[15] E. Huang, J. Crowcroft, and I. Wassell, "Rethinking incentives for mobile ad hoc networks," in Proc. Proceedings of the ACM SIGCOMM Workshop on Practice and Theory of Incentives in Networked Systems, PINS '04, ACM, New York, NY, USA, 2004, pp. 191-196. Article (CrossRef Link)

[16] Z. Song, C. H. Liu, J. Wu, J. Ma and W. Wang, "QoI-Aware Multitask-Oriented Dynamic Participant Selection With Budget Constraints," IEEE Transactions on Vehicular Technology, vol. 63, no. 9, pp. 4618-4632, Nov. 2014. Article (CrossRef Link)

[17] C. H. Liu, J. Fan, P. Hui, J. Crowcroft and G. Ding, "QoI-Aware Energy-Efficient Participatory Crowdsourcing," IEEE Sensors Journal, vol. 13, no. 10, pp. 3742-3753, Oct. 2013. Article (CrossRef Link)

[18] D. Zhao, H. Ma and L. Liu, "Frugal Online Incentive Mechanisms for Mobile Crowd Sensing," IEEE Transactions on Vehicular Technology, vol. 66, no. 4, pp. 3319-3330, April 2017. Article (CrossRef Link)

[19] Y. Lv, and T. Moscibroda, "Fair and resilient incentive tree mechanisms," in Proc. Proceedings of the 2013 ACM Symposium on Principles of Distributed Computing, PODC '13, ACM, New York, NY, USA, 2013, pp. 230-239. Article (CrossRef Link)

[20] B. Faltings, J. J. Li and R. Jurca, "Incentive Mechanisms for Community Sensing," IEEE Transactions on Computers, vol. 63, no. 1, pp. 115-128, Jan. 2014. Article (CrossRef Link)

[21] Y. Zhang and M. van der Schaar, "Reputation-based incentive protocols in crowdsourcing applications," 2012 Proceedings IEEE INFOCOM, Orlando, FL, pp. 2140-2148, 2012. Article (CrossRef Link)

[22] A. Albers, I. Krontiris, N. Sonehara, and I. Echizen, "Coupons as monetary incentives in participatory sensing," in Proc. Collaborative, Trusted and Privacy-Aware e/m-Services, Springer, 2013, pp. 226-237. Article (CrossRef Link)

[23] K. L. Huang, S. S. Kanhere, and W. Hu, "On the need for a reputation system in mobile phone based sensing," Ad Hoc Networks, Vol. 12, pp. 130 - 149, Jan. 2014. Article (CrossRef Link)

[24] Y. Zhang and M. van der Schaar, "Robust Reputation Protocol Design for Online Communities: A Stochastic Stability Analysis," IEEE Journal of Selected Topics in Signal Processing, vol. 7, no. 5, pp. 907-920, Oct. 2013. Article (CrossRef Link)

[25] Q. Li and G. Cao, "Providing privacy-aware incentives for mobile sensing," in Proc. of 2013 IEEE International Conference on Pervasive Computing and Communications (PerCom), San Diego, CA, pp. 76-84, 2013. Article (CrossRef Link)

[26] X. Oscar Wang, W. Cheng, P. Mohapatra and T. Abdelzaher, "ARTSense: Anonymous reputation and trust in participatory sensing," 2013 Proceedings IEEE INFOCOM, Turin, 2013, pp. 2517-2525. Article (CrossRef Link)

[27]X. Zhang, Z. Yang, Z. Zhou, H. Cai, L. Chen and X. Li, "Free Market of Crowdsourcing: Incentive Mechanism Design for Mobile Sensing," IEEE Transactions on Parallel and Distributed Systems, vol. 25, no. 12, pp. 3190-3200, Dec. 2014. Article (CrossRef Link)

[28] D. Zhao, X. Y. Li and H. Ma, "How to crowdsource tasks truthfully without sacrificing utility: Online incentive mechanisms with budget constraint," in Proc. IEEE INFOCOM 2014 - IEEE Conference on Computer Communications, Toronto, ON, pp. 1213-1221, 2014. Article (CrossRef Link)

[29] R. T. B. Ma, S. C. M. Lee, J. C. S. Lui and D. K. Y. Yau, "Incentive and Service Differentiation in P2P Networks: A Game Theoretic Approach," IEEE/ACM Transactions on Networking, vol. 14, no. 5, pp. 978-991, Oct. 2006. Article (CrossRef Link)

[30] W. S. Lin, H. V. Zhao and K. J. R. Liu, "Incentive Cooperation Strategies for Peer-to-Peer Live Multimedia Streaming Social Networks," IEEE Transactions on Multimedia, vol. 11, no. 3, pp. 396-412, April 2009. Article (CrossRef Link) 
[31] W. Tushar, W. Saad, H. V. Poor and D. B. Smith, "Economics of Electric Vehicle Charging: A Game Theoretic Approach," IEEE Transactions on Smart Grid, vol. 3, no. 4, pp. 1767-1778, Dec. 2012. Article (CrossRef Link)

[32] H. Park and M. van der Schaar, "Coalition-Based Resource Negotiation for Multimedia Applications in Informationally Decentralized Networks," in IEEE Transactions on Multimedia, vol. 11, no. 4, pp. 765-779, Jun. 2009. Article (CrossRef Link)

[33] D. Christin, A. Reinhardt, S. S. Kanhere, and M. Hollick, "A survey on privacy in mobile participatory sensing applications," Journal of Systems and Software, Vol. 84, Iss. 11, pp. 1928 1946, Nov. 2011. Article (CrossRef Link)

[34] Li Tianze, Wu Muqing and Wu Yuewei, "An game theory based online incentive mechanism for participatory sensing," in Proc. of 2016 7th IEEE International Conference on Software Engineering and Service Science (ICSESS), Beijing, pp. 700-705, 2016. Article (CrossRef Link)

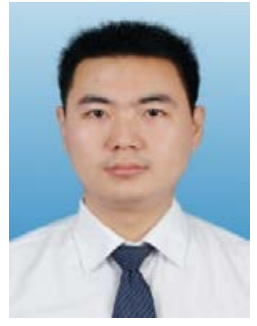

Hu Yao ( huyao800@gmail.com ) received his M.E degree in information and telecommunication systems from Chongqing University of Posts and Telecommunications (CQUPT), Chongqing, China in 2008. He is currently a Ph.D candidate in School of information and telecommunication, Beijing University of Posts and Telecommunications (BUPT), Beijing. His research interests are in the areas of congestion control and architecture design for data center networks.

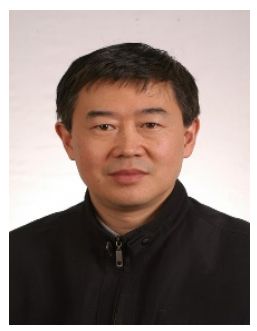

Wu Muqing born in July 1963, Ph.D, professor of Beijing University of Posts and Telecommunications (BUPT), senior membership of China institute of communications. Now, his research interests focus on mobile Ad Hoc network, UWB, high-speed network traffic control and performance analysis, GPS locating and services.

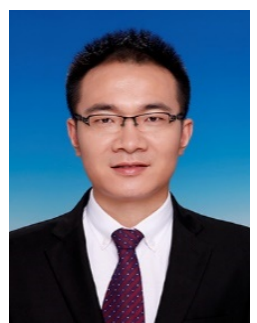

Li Tianze received his bachelor's degree in Applied Physics from Jiangnan University, Jiangsu, China in 2011. Since 2011 he studied in Beijing University of Posts and Telecommunications (BUPT) as a doctorate candidate. His research interests include the internet of things, Ad Hoc wireless network, wireless sensor network, participatory sensing, and mobile edge computing. 